\title{
Contribuições da atenção farmacêutica á pacientes em tratamento oncológico
}

\author{
Contributions of pharmaceutical care to patients on oncological treatment
}

\author{
Lívia Christina Almeida da Silva1; Poliana Oliveira Lemos de Brito2; Cristiane Dominice Melo, \\ Angela Falcai ${ }^{4}$;Ione Cristina de Paiva Pereira ${ }^{3}$
}

Resumo: Pacientes tratados com terapia antineoplásica são candidatos ao desenvolvimento de potenciais reações adversas devido a poliquimioterapia, margem estreita dos medicamentos em uso e tratamento prolongado. A Atenção Farmacêutica torna-se fundamental nesse processo, orientando o uso correto dos medicamentos, acompanhando reações adversas e interações medicamentosas, diminuindo o risco de erros e a descontinuidade do tratamento. O papel do farmacêutico no tratamento oncológico ainda é pequeno, mas vem evoluindo além da dispensação de medicamentos, esse profissional busca encontrar e resolver problemas relacionados a medicamentos que apareçam durante do tratamento. Portanto a inserção do farmacêutico na equipe multiprofissional do tratamento oncológico pode contribuir de forma positiva para o alcance da máxima efetividade e segurança da farmacoterapia e melhoria na qualidade de vida do paciente. Neste sentido o presente projeto tem por objetivo demonstrar a importância da Atenção Farmacêutica para a melhoria na qualidade de vida do paciente em tratamento antineoplásico e a necessidade de valorização e incorporação do profissional farmacêutico na equipe multidisciplinar.

Palavras chave: Atenção Farmacêutica. Farmacoterapia. Paciente oncológico

\begin{abstract}
Patients treated with antineoplastic therapy are candidates for the development of potential adverse reactions due to multidrug therapy, narrow margin of the drugs in use and prolonged treatment. Pharmaceutical Care is fundamental in this process, guiding the correct use of medicines, accompanying adverse reactions and drug interactions, reducing the risk of errors and discontinuation of treatment. The role of the pharmacist in cancer treatment is still small, but has evolved beyond the dispensing of medications, this professional seeks to find and solve problems related to drugs that appear during treatment. Therefore, the insertion of the pharmacist into the multiprofessional team of the cancer treatment can positively contribute to reach the maximum effectiveness and safety of the pharmacotherapy and improvement in the quality of life of the patient. In this sense, the present project aims to demonstrate the importance of Pharmaceutical Care for the improvement in the quality of life of the patient undergoing antineoplastic treatment and the need for valorization and incorporation of the pharmaceutical professional in the multidisciplinary team.
\end{abstract}

Keywords: Pharmaceutical care, pharmacotherapy, cancer patient

\footnotetext{
${ }^{1}$ Graduado no Curso de Farmácia da Universidade Ceuma. São Luís- MA, Brasil.

${ }^{2}$ Graduanda no Curso de Farmácia da Universidade Ceuma. São Luís-MA, Brasil.

${ }^{3}$ Docente do Curso de Farmácia da Universidade Ceuma .São Luís-MA, Brasil.

${ }^{4}$ Docente do Mestrado em Meio Ambiente - Universidade Ceuma. São Luís-MA, Brasil.
}

Autor Correspondente: Angela Falcai

Av. Deputado Luís Eduardo Magalhães, 1, Cond. Jardins Andaluzia, Torre Granada, Apto 204. Bairro Calhau. Cep 65071-415. São Luís - Maranhão. Email: afalcai@gmail.com 


\section{Introdução}

O Câncer é o nome geral dado a um grupo de patologias que têm em comum o crescimento desordenado de células que penetram os tecidos e órgãos, podendo espalhar-se para outras regiões do corpo. Estas células tendem a ser muito agressivas e incontroláveis, determinando a formação de tumores ou neoplasias malignas, porém, podem ser tratadas e seus índices de cura atualmente são muito elevados. Outra característica que diferenciam os diversos tipos de câncer é, a velocidade de multiplicação das células. ${ }^{1}$

O número de casos de câncer tem aumentado de maneira considerável em todo mundo. A Organização Mundial de Saúde - OMS (2012) fez uma projeção de 27 milhões de novos casos de câncer até 2030 em todo o mundo e 17 milhões de mortes pela a doença. Segundo dados do INCA ${ }^{2}$, os países em desenvolvimento serão os mais afetados, entre eles 0 Brasil. Dados epidemiológicos revelam que só no ano de 2016 foram registrados no país 596 mil novos casos de câncer, os tipos com maior incidência são o câncer de pele, próstata e mama (INCA, 2016). ${ }^{2}$

Os fármacos utilizados na terapia antineoplásica têm a capacidade de impedir o desenvolvimento de processos vitais das células tumorais, porém não possuem uma especificidade para estas células, podendo agir também em células saudáveis do organismo, desse modo muitos medicamentos apresentam uma janela terapêutica estreita, ou seja, a dose usual é próxima a dose tóxica e muitos também podem ser classificados carcinogênicos. Geralmente as reações adversas predominantes neste grupo de medicamentos estão relacionados, náuseas e vômitos, supressão da medula óssea e alopecia, além de toxicidade pulmonar, neurotoxicidade, toxicidade renal, cardiotoxicidade, lesão godonal e esterilidade. Vale destacar que os efeitos adversos são um fator que influencia para não adesão à terapia pelo paciente e podem interferir na efetividade, provocando um avanço da doença. ${ }^{3}$

A terapia do paciente oncológico necessita de diversos tratamentos combinados, sendo relevante um acompanhamento individualizado e especializado, considerando as suas necessidades. Nesse contexto, é de suma importância que a equipe multiprofissional faça a orientação adequada ao paciente durante todo o tratamento. Essa equipe é composta por médicos, enfermeiros, psicólogos, nutricionistas, entre outros profissionais. ${ }^{4}$ Mais recentemente, o farmacêutico ocupou seu espaço nessa equipe de profissionais, tornando-se indispensável para a qualidade do processo farmacoterapêutico.

O acompanhamento do farmacêutico aos pacientes em tratamento oncológico é uma importante ferramenta para a redução de erros de medicação no tratamento, tornando-o mais eficaz e melhorando a qualidade de vida, sua tarefa é garantir que a terapia medicamentosa do doente esteja devidamente adequada e que seja a mais segura e conveniente ao paciente. Neste sentido a Atenção Farmacêutica pode ter um papel importante neste processo. ${ }^{5}$

A Atenção Farmacêutica é uma prática profissional voltada para o paciente onde 0 farmacêutico fará 0 aconselhamento e monitoramento da terapia farmacológica em que o paciente está inserido, prestando todas as informações necessárias em relação ao medicamento para garantir a adesão ao tratamento e uso racional do medicamento. Por meio desta atenção o farmacêutico torna-se co-responsável pela qualidade de vida do paciente. ${ }^{6}$

O farmacêutico desempenha um importante papel para a edificação de um novo modelo de atenção à saúde, onde ele possa estar colocado como profissional do medicamento, atuando como referência na orientação, 
cumprimento, acompanhamento e monitoramento da terapia farmacológica.

A presente pesquisa demonstrar as contribuições da Atenção Farmacêutica para pacientes oncológicos ressaltando a importância e necessidade da atuação do farmacêutico no acompanhamento e monitoramento da terapia farmacológica.

\section{Material e método}

Trata-se de uma pesquisa bibliográfica descritiva com 0 intuito identificar publicações a respeito das contribuições da Atenção Farmacêutica ao paciente oncológico em tratamento, baseada em pesquisas realizadas no período de 2007 a 2017, utilizando-se artigos e teses relacionados ao tema proposto. Para localizar publicações será realizada uma busca nas bases de dados on-line BVS (Biblioteca Virtual em Saúde), Scielo. Google acadêmico e Pubmed nos meses de setembro de 2016 a abril de 2017.

\section{Resultados e discussão}

A atuação do farmacêutico em oncologia é uma realidade presente em praticamente todos os serviços de quimioterapia. Embora tenha iniciado sua atuação exclusivamente nas atividades de manipulação e gerenciamento de quimioterápicos, tornou-se peça fundamental para a garantia de qualidade dos procedimentos. ${ }^{7}$ Além das atribuições relacionadas ao preparo da terapia antineoplásica, cabe ao farmacêutico compor a equipe multiprofissional nas visitas aos pacientes submetidos ao tratamento oncológico. ${ }^{8}$ Conhecer os aspectos farmacológicos, suas propriedades, mecanismos e efeitos adversos dos medicamentos, é o principal fator para o sucesso e qualidade da farmacoterapia de um paciente, uma vez que faz do farmacêutico uma peça fundamental em todo o processo de tratamento. ${ }^{7}$

Há anos o farmacêutico vem ampliando a sua atuação no universo da oncologia, desde que o Conselho Federal de Farmácia estabeleceu que é uma função privativa do farmacêutico a competência para o exercício da atividade de manipulação de drogas antineoplásicas e similares nos estabelecimentos de saúde, e no exercício desta atividade tendo também outras atribuições relacionadas. ${ }^{8}$

Diante do crescimento no número de pacientes oncológicos inseridos em protocolos onde há necessidade de terapia antineoplásica, se faz necessário o esclarecimento sobre os medicamentos ao paciente. ${ }^{9}$ Logo, o farmacêutico é um profissional indispensável na equipe multiprofissional do tratamento oncológico, qualificado a desenvolver várias funções dentro da equipe multidisciplinar, como Atenção Farmacêutica aos pacientes oncológicos e informações aos demais profissionais da equipe de saúde ${ }^{10-11}$.

Assim, o farmacêutico vai agregando atitudes, valores éticos, habilidades, responsabilidade na prevenção de doenças e recuperação da saúde com a integração da equipe. É a relação mais próxima do farmacêutico com o usuário de medicamentos, levando a uma farmacoterapia racional com a obtenção de resultados voltados a melhoria da qualidade de vida. ${ }^{12}$ Os pacientes se sentem amparados quanto às informações relacionadas à ação dos fármacos, seus efeitos adversos, às interações medicamentosas e ao desenvolvimento do tratamento, podendo contribuir significativamente para o seu sucesso, até porque muitos pacientes relutam em aderir a um tratamento, pois não foram esclarecidos devidamente. ${ }^{13}$

O processo de atenção farmacêutica começa quando o paciente disponibiliza informações a respeito do seu tratamento, sendo que as primeiras referências são coletadas no prontuário 
médico e comprovadas por meio de entrevista com o paciente, permitindo que o farmacêutico analise a indicação e a posologia de cada medicamento em uso, averiguando interações medicamentosas, condições de armazenamento e verificando problemas relacionados aos medicamentos. ${ }^{7}$ É uma atividade específica do farmacêutico no cuidado do paciente ou usuário de medicamento. ${ }^{14}$ Compreende a educação em saúde, orientação farmacêutica, dispensação, atendimento farmacêutico, acompanhamento farmacoterapêutico, registro sistemático das atividades, determinação e estimativa dos resultados. O farmacêutico deve relacionar-se de maneira ativa com 0 paciente buscando solucionar problemas que envolvam ou não o uso de medicamentos e acompanhar seus resultados, para que desta forma, a dispensação do medicamento ao paciente seja feita de forma consciente e segura. ${ }^{15}$

Os eventos adversos da terapia oncológica são conhecidamente agressivos aos pacientes o que pode ocasionar o abandono da terapia ou também a diminuição na qualidade de vida, pelas dificuldades físicas e psicológicas que surgem no decorrer de todo 0 tratamento. A atenção farmacêutica ao paciente oncológico requer do profissional farmacêutico atributos mais específicos, que não somente abordem o conhecimento da detecção de problemas associados aos medicamentos, mas também adentrem o campo do aconselhamento nãofarmacológico, com a utilização de linguagem simples e próxima da realidade do paciente, ao ponto que este possa entender e contornar os eventos adversos de forma mais amena ${ }^{9}$.

Por meio de recursos utilizados pelo farmacêutico na análise de prescrição médica, a terapia antineoplásica torna-se mais segura para o paciente ${ }^{7}$. Esta é uma das principais atividades do farmacêutico clínico, pois com 0 prontuário nas mãos e o conhecimento clínico do paciente é possível fazer o acompanhamento farmacoterapêutico analisando a prescrição quanto à dose dos medicamentos, diluição e tempo de infusão, via e frequência de administração, compatibilidade e interações. $^{16} \quad \mathrm{O}$ acompanhamento farmacoterapêutico é um grande desafio para o profissional farmacêutico e se torna uma ferramenta importante para reduzir os erros com medicações, o que implica na eficácia do tratamento e na melhora da qualidade de vida. ${ }^{17}$ Cabe ao farmacêutico desenvolver e utilizar estratégias para favorecer a comunicação com o paciente a fim de realizar 0 acompanhamento farmacoterapêutico. ${ }^{18}$

O tratamento farmacológico deverá ser adequado à forma de vida de cada paciente, considerando suas limitações, hábitos, sua motivação para cumprir o plano terapêutico, objetivando garantir a adesão ao tratamento e melhorar a qualidade de vida do paciente. Trata-se, portanto, de uma conquista facilitada pela cumplicidade desenvolvida entre farmacêutico e paciente. ${ }^{19} \mathrm{O}$ cuidado farmacêutico não envolve apenas terapia medicamentosa, mas também envolve decisões sobre 0 uso adequado de medicamentos para cada doente como por exemplo a seleção da dose e via de administração, a oncologia desenvolvese, de forma muito dinâmica e o farmacêutico é desafiado a manter-se informado sobre as novas terapias. ${ }^{20-21}$

Em contrapartida Suzuki ${ }^{22}$ em um estudo realizado no Japão com 583 farmacêuticos, para avaliar o seu nível de segurança em prestar esclarecimentos sobre o tratamento antineoplásicos aos pacientes em tratamento, verificou que apenas $6-10 \%$ dos farmacêuticos achavam que tinham recebido uma educação adequada sobre quimioterapia e, portanto, não se sentiam seguros o suficiente para prestar atenção farmacêutica na oncologia. Embora 81\% deles, tenham participado de 
treinamentos continuados relacionados à oncologia, somente $54 \%$ se sentiam confortáveis em dispensar agentes anticancerígenos orais e apenas $40 \%$ em educar os pacientes sobre o tratamento.

Por outro lado, os bons resultados da Atenção Farmacêutica em pacientes sob tratamento oncológico já foram verificados e atestados por vários estudos na área, entre eles, o de Nightingale ${ }^{23}$, nos Estados Unidos, para testar a viabilidade e eficácia da implementação da Atenção Farmacêutica individualizada. $\mathrm{O}$ estudo avaliou 48 pacientes em tratamento quimioterápico e identificou que $95 \%$ destes pacientes apresentavam problemas relacionados a medicamentos. Além disso, o estudo verificou ainda que a Atenção Farmacêutica contribuiu para redução média de $45,5 \%$ destes problemas no prazo de 60 dias, concluindo que tal atividade contribui de forma significativa para redução dos problemas relacionados a medicamentos.

$\mathrm{Na}$ Espanha, 102 pacientes participaram de um estudo realizado por Caracue ${ }^{24}$ para analisar os efeitos da Atenção Farmacêutica sobre a incidência de reações adversas a medicamentos, principalmente náuseas e vômitos induzidos pela quimioterapia e foi observado que com um acompanhamento farmacoterapêutico houve uma redução de $59 \%$ destas reações, 0 que consequentemente melhorou a adesão ao tratamento.

Outro estudo realizado na Holanda, por Lopez ${ }^{25}$ para verificar a incidência de interações medicamentosas em pacientes que recebiam quimioterapia e investigar a influência das intervenções farmacêuticas durante 0 tratamento, constatou que o farmacêutico conseguiu intervir em 20 casos onde foi identificado algum tipo de interação medicamentosa, o que ajudou a descontinuar ou modificar as prescrições solucionando assim, 94\% dos casos.

$\mathrm{Chew}^{26}$, em uma pesquisa realizada em Cingapura, para identificar a taxa de aceitação das intervenções farmacêuticas em solucionar problemas relacionados a medicamentos, registrou um total de 331 intervenções farmacêuticas e uma taxa de $93 \%$ de aceitação, demonstrando que esta atividade proporciona resultados positivos para a terapia de cada paciente.

No Brasil foi realizado no Rio de Janeiro no Hospital do Câncer I - INCA por Couto ${ }^{27}$ um Projeto Piloto de Atenção Farmacêutica, onde foram realizadas intervenções farmacêuticas em 23 pacientes e houve uma aceitação de $81 \%$ dos casos, sendo que o farmacêutico contribuiu para resolução de $79 \%$ dos problemas relacionados a medicamentos. $\mathrm{O}$ projeto trouxe resultados positivos $\mathrm{e}$ significativos resultando na sua implantação permanente.

$\mathrm{Na}$ farmácia hospitalar, o papel desempenhado pelo farmacêutico nas etapas de seleção, aquisição, armazenamento, controle e distribuição de medicamentos já é amplamente conhecido. No entanto 0 seu envolvimento com a farmácia clínica vem possibilitando uma participação mais efetiva no processo de acompanhamento farmacoterapêutico e aproximação do paciente. ${ }^{28}$

A atuação do farmacêutico clínico, através do trabalho de atenção farmacêutica, junto à equipe multiprofissional, visa promover a qualidade da terapêutica do paciente, uma vez que orienta os profissionais sobre o uso seguro e racional dos medicamentos. Este profissional é importante na identificação, correção e redução de possíveis riscos associados à terapêutica, sendo o paciente o principal beneficiário das suas ações ${ }^{29}$, compete a ele acompanhar diariamente o trabalho da equipe buscando agregar seus conhecimentos farmacológicos na qualidade do trabalho assistencial ${ }^{16}$. A Farmácia Clínica pressupõe que o farmacêutico estabeleça um relacionamento ativo com os demais membros da equipe de saúde, 
principalmente médicos e enfermeiros. Porém, também pressupõe o contato com os pacientes, para assim assegurar resultados clinicamente apropriados para a farmacoterapia, o paciente é objeto principal das atividades do farmacêutico hospitalar. ${ }^{30}$

O paciente oncológico sofre desde 0 diagnóstico da doença, passando pelo tratamento e também o período pós tratamento, enfrentando momentos de ansiedade e stress, ele se torna mais vulnerável e carente, merecendo carinho e atenção especial por parte de todos os integrantes da equipe de saúde. $A$ presença do farmacêutico oferecendo informações adequadas ameniza a preocupação do paciente, transmitindo mais segurança, prevendo e tratando possíveis reações adversas e, portanto, melhorando os resultados obtidos com a terapia. $^{5}$

\section{Conclusão}

A prática da Atenção Farmacêutica ao paciente oncológico em tratamento é possível devido a necessidade de detectar possíveis suspeitas de problemas relacionados a medicamentos, a fim de buscar maneiras de amenizar reações adversas que acometem a grande maioria dos pacientes que estão em tratamento com antineoplásicos, visando a melhoria na qualidade de vida do paciente e uma terapia segura. Aproximando o profissional farmacêutico do paciente, muda-se a postura comumente empregada no ambiente, seja ele hospitalar ou ambulatorial, o farmacêutico passa a enxergar o paciente como foco de seu trabalho.

Baseado no levantamento de dados dos artigos selecionados observou-se que a prática da atenção farmacêutica é uma atividade promissora por inúmeras razões: acessibilidade; redução de custos; melhor acompanhamento e eficácia do tratamento farmacológico; uso racional dos medicamentos; redução de problemas relacionados a medicamentos (PRM); melhoria na qualidade de vida; contribuição para adesão ao tratamento; melhoria na relação farmacêutico/paciente. Ressaltando que todas as contribuições da desta atividade são para benefício exclusivo do paciente.

Desta forma conclui-se que a participação ativa do farmacêutico junto aos pacientes em tratamento e junto à equipe multiprofissional se faz necessária, pois este profissional possui qualificações para desempenhar na oncologia papel administrativo e clínico cooperando para uma terapia segura aos pacientes em tratamento e também com os membros da equipe. 


\section{Referências}

1. Instituto Nacional do Câncer José Alencar Gomes da Silva. O Câncer e Seus Fatores de Risco. O que a educação pode evitar? Rio de Janeiro: INCA, 2013.

2. Instituto Nacional do Câncer José Alencar Gomes da Silva. Estimativa 2016: Incidência de câncer no Brasil. Rio de Janeiro: INCA; 2015.

3. MS, Abreu PA, Nogueira TA. Atenção Farmacêutica a Pacientes Oncológicos: Uma Revisão Integrativa da Literatura. Scielo. 2016; 7.

4. Almeida JRCD. Farmacêuticos em Oncologia, uma Nova Realidade. $2^{\text {nd }}$ ed. São Paulo (BR): Atheneu; 2010.

5. Leão AMD. et al. Atenção Farmacêutica no Tratamento Oncológico em um Instituição Pública de Montes Claros - MG. Scielo. 2012; 3.

6. Silva GADO. Farmacêutico na Vida do Paciente com Câncer [Internet]. Portal da Educação; 2012 [acesso em 2016 nov]. Disponivel em: www.portaldaeducação.com.br/conteu do/artigos/nutricao/atuacao-dofarmaceutico-no-tratamento-docancer/20979.

7. ESCOBAR, Graziela. Um novo modelo para a oncologia. Newsletter científico do Centro de Combate ao câncer, São Paulo, ed.1 n. 01 p. 1-2, janeiro 2010.

8. BRASIL. Resolução 565 de 06 de dezembro de 2012 - Dispõe sobre a competência legal para atuação do farmacêutico nos serviços oncológicos, 2012.

9. Oliveira ATD.; DE ALMEIDA QUEIROZ, A. P. Perfil de uso da terapia antineoplásica oral: a importância da orientação farmacêutica. Scielo. 2012; 3.

10. Leão AMD. et al. Atenção Farmacêutica no Tratamento Oncológico em um Instituição Pública de Montes Claros - MG. Scielo. 2012; 3.
11. Matile E. Papel do Farmacêutico na Oncologia: da manipulação à Assistência Farmacêutica. Revista Brasileira de Oncologia. 2008; 5.

12. Oliveira PVD. O farmacêutico em oncologia - o que temos, podemos e fazemos [monografia]. Araraquara: Universidade Estadual Paulista UNESP; 2013.

13. Moreira RB, Boechat, L. Proposta de Acompanhamento

Farmacoterapêutico em Leucemia Mielóide Crônica: Modelo de Abordagem Metodologica. Revista Brasileira de Cancerologia. 2009; 4.

14. Angonesi D, Sevalho G. Atenção Farmacêutica: fundamentação conceitual e crítica para um modelo brasileiro. Ciência e Saúde Coletiva. 2010; 15.

15. Pinho MS, Abreu PA, Nogueira TA. Atenção Farmacêutica a Pacientes Oncológicos: Uma Revisão Integrativa da Literatura. Scielo. 2016; 7.

16. Ferracini FT, Filho WBM. Farmácia Clínica, segurança na prática hospitalar. São Paulo (BR): Atheneu; 2012.

17. Sturaro D. A importância do acompanhamento farmacoterapêutico em pacientes onco-hematológicos. Rev. Brasileira de Hematologia. 2009; 3.

18. Possai, F. P.; Dacoreggio, M. S. A habilidade de comunicação com 0 paciente no processo de Atenção Farmacêutica. Trab. Edu. Saúde, v. 5, 2008.

19. Ribeiro VF. et al. Realização de Intervenções Farmacêuticas por meio de uma experiência em Farmácia Clínica. Scielo. 2015; 6.

20. Sousa RICMD. Cuidados Farmacêuticos no Doente Oncológico [monografia]. Licenciatura em Ciências Farmcêuiticas. Porto: Universidade Fernando Pessoa; 2010.

21. Andrade CC. Farmacêuticos em Oncologia: interfaces administrativas 
e clínicas. Pharmacia Brasileira. 2009; 1-24.

22. Suzuki S. et al. Evaluation of community pharmacist ability to ensure the safe use of oral anticancer agents: a nationwide survey in Japan. JJCO. 2017; 47(5).

23. Nightingale $G$. et al. Implementing a pharmacist-led, individualized medication assessment and planning (iMAP) intervention to reduce medication related problems among older adults with cancer. Elsevier. 2017.

24. Caracuel F. et al. Influence of pharmaceutical care on the delayed emesis associated with chemotherapy. MEDLINE. 2014; 36.

25. Lopez CM. et al. Role of clinical phamacistis in preventing drug interações in out patients with cancer: a unique experience. MEDLINE. 2014.

26. Chew C, Chiang J, Yeoh T. Impact of out patients interventions done at an out patient cancer oncology pharmacy center in Singapore. 2015.

27. Couto DHN. et al. Projeto Piloto de Atenção Farmacêutica no Hospital do Câncer I/INCA. 2010.

28. Souza JAADOE, Cordeiro BC. Atenção Farmacêutica às Pacientes Oncológicas de um Hospital de Grande Porte do Rio de Janeiro. Scielo. 2012; 3.

29. Ribeiro MAS. et al. Farmacêutico na oncologia: interfaces administrativas e clínicas - Pharmacia Brasileira. Comissão de Farmácia Hospitalar do CFF - Conselho Federal de Farmácia. 2009.

30. Storpitis S. Farmácia Clínica e Atenção Farmacêutica. Rio de Janeiro: Guanabara; 2008. 